\title{
Assessing the effect of beard hair lengths on face masks used as personal protective equipment during the COVID-19 pandemic
}

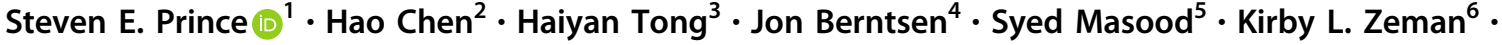 \\ Phillip W. Clapp ${ }^{6,7} \cdot$ William D. Bennett ${ }^{6,8} \cdot$ James M. Samet $^{3}$
}

Received: 25 January 2021 / Revised: 14 April 2021 / Accepted: 22 April 2021 / Published online: 18 May 2021

This is a U.S. government work and not under copyright protection in the U.S.; foreign copyright protection may apply 2021

\begin{abstract}
Background Globally, a large percentage of men keep a beard at least occasionally. Workplace regulations prohibit beards with N95 respirators, but there is little information on the effect of beards with face masks worn by the public for protection against SARS-CoV-2.

Methods and findings We examined the fitted filtration efficiency (FFE) of five commonly worn protective face masks as a function of beard length following the US Occupational Safety and Health Administration Quantitative Fit Test: N95 (respirator), KF94 and KN95, surgical/procedure, and cloth masks. A comparison using N95 respirators was carried out in shaven and bearded men. A detailed examination was conducted for beard lengths between 0 and $10 \mathrm{~mm}(0.5 \mathrm{~mm}$ increments). The effect of an exercise band covering the beard on FFE was also tested. Although N95 respirators showed considerable variability among bearded men, they had the highest FFE for beard lengths up to $10 \mathrm{~mm}$. KF94 and KN95 masks lost up to $40 \%$ of their FFE. Procedure and cotton masks had poor performance even on bare skin (10-30\% FFE) that did not change appreciably with beard length. Marked performance improvements were observed with an exercise band worn over the beard.

Conclusions Though variable, N95 respirators offer the best respiratory protection for bearded men. While KF94 and KN95 FFE is compromised considerably by increasing beard length, they proved better options than procedure and cotton face masks. A simple exercise band improves FFE for face masks commonly used by bearded men during the COVID-19 pandemic.
\end{abstract}

Keywords mask $\cdot$ respiratory protection $\cdot \mathrm{SARS}-\mathrm{CoV}-2 \cdot$ beard $\cdot$ intervention $\cdot$ particles

These authors contributed equally: Steven E. Prince, Hao Chen

Supplementary information The online version contains supplementary material available at https://doi.org/10.1038/s41370021-00337-1.

Steven E. Prince

Prince.Steven@epa.gov

1 Public Health and Environmental Systems Division, Center for Public Health and Environmental Assessment, Office of Research and Development, US Environmental Protection Agency, Research Triangle Park, NC, USA

2 Oak Ridge Institute for Science Education, Oak Ridge, TN, USA

3 Public Health and Integrated Toxicology Division, Center for Public Health and Environmental Assessment, Office of Research and Development, US Environmental Protection Agency, Research Triangle Park, NC, USA

4 TRC, Raleigh, NC, USA

\section{Introduction}

The broad variety of face masks of varying efficiencies currently in use by the public represent a primary preventive measure to control the transmission of the SARS-CoV-2

5 Curriculum in Toxicology and Environmental Medicine, University of North Carolina at Chapel Hill, Chapel Hill, NC, USA

6 Center for Environmental Medicine, Asthma and Lung Biology, University of North Carolina at Chapel Hill, Chapel Hill, NC, USA

7 Department of Pediatrics, University of North Carolina at Chapel Hill, Chapel Hill, NC, USA

8 Department of Medicine, University of North Carolina at Chapel Hill, Chapel Hill, NC, USA 
virus as an intervention [1]. While the availability of highefficiency N95 respirators has been largely limited to health care professionals due to supply shortages, Chinese (KN95) and Korean (KF94) designed face masks, surgical-style disposable masks, and reusable cloth masks of widely varying material, design, and construction are currently in use by the general public.

A number of intrinsic factors influence the filtration performance of a face mask. Properties such as the porosity and surface charge of the face mask material can be seen as representing the theoretical limit of the efficiency of a face mask. On the other hand, design features influence the extent to which this limit can be approximated by determining the integrity of the seal that can be achieved and maintained between the face mask material and the facial skin. Extrinsic factors linked to the wearer that are known to affect face mask performance include variations in facial morphology [2] and the presence of facial hair [3].

According to a 2017 survey, approximately a third of adult males in the US report keeping a beard, with another $27 \%$ responding that they do so sporadically [4] and in a 2016 British survey, 37\% of all males reported beards or stubble, with $61 \%$ of 18 - to 39 -year olds reporting some form of facial hair [5]. Relative to bare skin, facial hair potentially alters the fit and performance of a face mask by changing the texture of the skin as well as topology at the points of contact. With the exception of a few styles, regulations established by the US Occupational Safety and Health Administration (OSHA) disallow facial hair for the workplace use of respirators such as N95 filtering face pieces [3]. However, information on the effect of facial hair on the efficacy of other types of face masks is lacking.

Guidance and regulations from government agencies, including the UK Health and Safety Executive [6], US Centers for Disease Control and Prevention/National Institute for Occupational Safety and Health [7], and the US OSHA [3] describe facial hair, stubble, and beards in particular, as a contraindication to the workplace use of tightfitting respirator masks. OSHA respiratory standards, finalized in 1998, describe beard growth as one condition that "interferes with the face-to-facepiece seal." Since the 1980s, several studies that examined the impacts of beard hair on standardized respirator masks provided the basis for agency guidelines. Although one study examining workplace protection factors found no clear relationship, multiple studies provided evidence for beards causing an impaired seal, reduced fit factors and detrimental impacts on respirator performance. This led agencies to advise against bearded workers using these respirator masks (in a workplace setting) and in the US to prohibit their use and fit testing for bearded workers. Suggested remedies for this include the use of loose-fitting hoods or helmets that would accommodate facial hair. Current guidance on the issue of beard hair is highly specific to the use of tight-fitting respirators in the workplace and there is a lack of information on costeffective face masks that are available to the general public during the COVID-19 pandemic or practical solutions for usage scenarios in the general public.

Given the importance of face masks as a primary preventive measure in controlling the spread of the SARSCoV-2 virus, understanding the factors that both harm and help their filtering performance is critical to public health. In the current study, we examine in detail the relationship between varying lengths of beard hair and the fitted filtration efficiency (FFE) of a variety of face masks used to prevent SARS-CoV-2 infection during the COVID-19 pandemic, and identify a potential solution to overcome decrements in FFE caused by the presence of beard hair.

\section{Methods}

\section{Testing procedure}

All face masks were fitted on adult male staff members $(n=10)$ who volunteered to be tested using the US Occupational Safety and Health Administration Quantitative Fit Testing Protocol (Modified Ambient Aerosol CNC Quantitative Fit Testing Protocol For Filtering Facepiece Table A-2-RESPIRATORS). Conditions related to the spread of SARS-CoV-2 necessitated modified recruitment and testing of volunteers. The aerosol filtration efficiency tests were conducted in a custom-built $10 \times 10$ foot stainless-steel exposure chamber (US EPA Human Studies Facility, Chapel Hill, NC) as recently described [8]. Briefly, a TSI 8026 Particle Generator (TSI, Inc., Shoreview, MN) was used to supplement the chamber with $\mathrm{NaCl}$ particles with a median diameter of $0.05 \mu \mathrm{m}$, as measured by a scanning mobility particle sizer (MODEL 3938 TSI, Inc). Prior to testing, the test atmosphere was allowed to stabilize for $30 \mathrm{~min}$ in an atmosphere in which the temperature and humidity during testing ranged from 21.5 to $25^{\circ} \mathrm{C}$ and 32 to $55 \%$, respectively. A steel sampling port was installed in each mask using a TSI model 8025-N95 Fit Test Probe Kit to allow sampling behind the mask. A pair of TSI 3775 condensation particle counters (CPCs) was run in single particle analysis mode to continuously monitor ambient particles in the size range $0.02-3 \mu \mathrm{m}$ in the chamber air just outside the face mask and particles in the breathing space behind the face mask at a sampling rate of $1 \mathrm{~s}$. Ten feet of 0.25 -inch conductive rubber tubing was used for each sampling line and was connected to the face mask through metal Luer connector fittings. The ambient sampling line and masks sampling lines were made identical to reduce variability in the system. Chamber particle counts/cc (cubic centimeter) reported by the CPCs were typically in the range of 
$6000-9000$, meaning that $95 \%$ reduction results in counts of $300-450$ or less inside the mask, with enough instrument dynamic range to accurately detect filtration performance in excess of $99 \%$. The overall mean filter efficiency was averaged from start to end of the testing period, and the average standard deviation over the period of sampling was computed.

The FFE of the face mask was measured while the subject performed a series of repeated movements of the torso, head, and facial muscles (i.e., bending at the waist, reading aloud, looking left and right, and looking up and down) designed to simulate workplace activities, as prescribed by the OSHA Fit Test. Although related metrics such as particle penetration or fit factors are also found in the literature, FFE is reported here as a commonly understood value of mask or respirator performance. The FFE $(\%)$ is calculated as $[1-($ mask count/ambient count $)] \times 100$ (the concentration of particles behind the mask divided by the particle concentration in the chamber atmosphere). Data were collected over the duration of each test described in the OSHA protocol (approximately $140 \mathrm{~s}$ plus $10 \mathrm{~s}$ between exercises).

\section{Face masks tested}

Five face masks representative of respiratory protection options available to the public during the COVID-19 pandemic were selected for testing: an N95 respirator (Model \#8210 TC 84A-0007, 3M St. Paul, MN), a KN95 earloop mask (Lei Shi De, EN149-2001+A1:2009, CIRS Garments, Shandong, PRC), a KF94 mask worn with supplied clip affixed to ear loops in the back of the head (Dr Puri, KM Corporation, Gyeonggi-do, Republic of Korea), an earloop procedure mask (Medline Industries, Northfield, IL), and a reusable 3-ply $100 \%$ cotton fabric face mask with ear loops and adjustable nosepiece (Hanesbrands, WinstonSalem, NC).

\section{Testing procedures}

All bearded volunteers $(n=5)$ had full facial hair that covered the jaw and cheeks, with starting lengths varying between 9 and $30 \mathrm{~mm}$. Beards tested all featured hair that extended into the respirator sealing surface area and, therefore, are contraindicated for respirator use by the National Institute of Occupational Safety and Health guidance. Two volunteers additionally trimmed their beards to varying lengths. Volunteer 1 (head circumference $55.9 \mathrm{~cm}$ ), who repeated testing 2-3 times for each length (in both ascending and descending growth across multiple testing days), used a Phillips Norelco QT4018 (Norelco, Andover, MA) beard trimmer equipped with length adjustments between 0.5 and $10 \mathrm{~mm}$, with $0.5 \mathrm{~mm}$ increments.

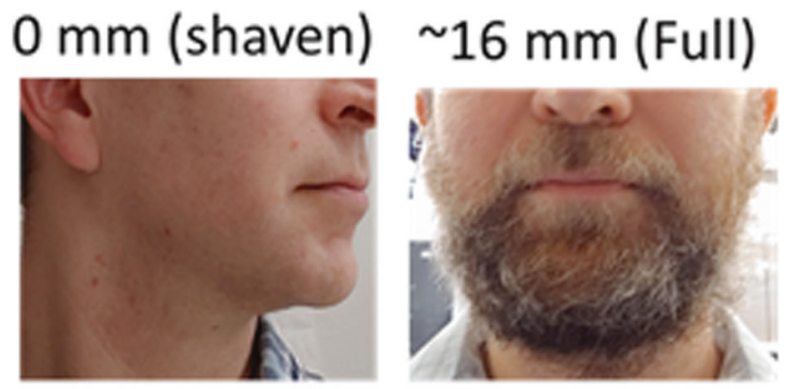

Green / Blue Exercise Band
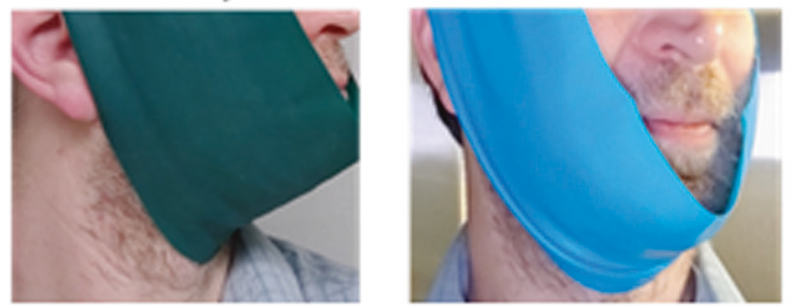

Fig. 1 Shaven, bearded and beard-covered conditions tested in this study. Volunteer 1 shown within 1 hour of a shave (top left panel), with the maximal beard length tested (top right panel), and wearing non-latex exercise (yoga) bands of light (bottom left) and medium (bottom right) resistance as a beard hair cover.

Volunteer 2, with a head circumference of $57.4 \mathrm{~cm}$, used a multifunction trimmer (Model SH1970, GOLEEN, Guangdong, PRC) fitted with 9-, 6-, and 3-mm combs in a descending order in a single day (data from three face masks shown in Supplementary Fig. 1). Volunteers with no facial hair $(n=5)$ used disposable razors and shaving cream to shave within $6 \mathrm{~h}$ of testing. Volunteer 1 also tested an intervention strategy using two different yoga style nonlatex exercise resistance bands ("light" green color 60" length $\times 4$ " width (Theraband, Akron, $\mathrm{OH}$ ), and "medium" blue color 60" length $\times 6$ " width (A Azurelife, Hong Kong)) worn over the beard. Prior to testing, the band was wrapped around the beard and knotted at the top and middle of the head, leaving access to properly affix all masks. Figure 1 shows Volunteer 1 shaven, with a full beard and with the exercise bands tested; see Supplementary Fig. 2 for complete photos of all beard lengths. Informed consent was obtained to publish these images in an online open access publication.

\section{Results}

A preliminary fit test survey of N95 respirator performance showed overall high FFE when worn by bearded men who were trained in proper donning and were supervised during the process. As shown in Table 1, the presence of a beard with an average hair length between 9 and $30 \mathrm{~mm}$ appeared to have only minor impact overall, when compared to FFE 
Table 1 N95 respirator fitted filtration efficiency (FFE) against submicron particles for shaved and unshaven men.

\begin{tabular}{lll}
\hline Subject & Average beard length $(\mathrm{mm})$ & $\begin{array}{l}\% \text { FFE }(\mathrm{SD})^{\mathrm{a}} \\
\text { N95 }\end{array}$ \\
\hline 1 & 0 & $99.4(0.5)$ \\
2 & 0 & $99.3(0.4)$ \\
3 & 0 & $99.1(0.3)$ \\
4 & 0 & $99.0(0.5)$ \\
5 & 0 & $97.6(1.8)$ \\
Average & & 98.9 \\
6 & 9 & $96.9(1.1)$ \\
7 & 9.8 & $97.1(1.0)$ \\
8 & 11.2 & $98.4(0.4)$ \\
9 & 15.7 & $85.0(3.0)$ \\
10 & 30.4 & $98.8(0.4)$ \\
Average & & 95.2
\end{tabular}

${ }^{\mathrm{a}}$ The efficiency is calculated as [1-(mask count/ambient count $\left.)\right] \times 100$ across the length of the test. The FFE \pm SD are shown.

in shaven men. Volunteer 1, the bearded staff member who showed the lowest FFE with an N95 respirator, underwent additional fit testing at varying beard lengths, and with additional face mask types that are available to the public during the COVID-19 pandemic.

Figure 2 shows FFE (\%), sampled at 1-s intervals, during different phases of fit testing (i.e., bending at the waist, reading aloud, turning of head, raising, and lowering of head) with an N95 respirator, KF94, KN95, a procedure style mask, and a 3-ply $100 \%$ cotton mask, as a function of beard lengths between 0 (shaven), 1.5, 3, 6, 9, and $>10 \mathrm{~mm}$. Results show averaged values across three unique tests. A repeating pattern, conserved across nearly all face masks tested, was observed in which FFE was highest during reading aloud (i.e., while exerting a positive pressure inside the mask). Interestingly, exceptions to this pattern were for the N95 respirator between 0 and $2.5 \mathrm{~mm}$ of beard length (Fig. 2A-E and Supplementary Tables 1-5). The rapid inhalation and facial movements associated with speech likely have an effect of pulling a looser fitting mask or respirator toward the face, versus challenging the seal when a more rigid respirator fits tightly.

To generate a more detailed view of beard length as determinant of face mask performance, we retested the face masks' FFE for Volunteer 1 at $0.5 \mathrm{~mm}$ increments over a range of $0-10 \mathrm{~mm}$ (Fig. 3). Three additional measurements at an average hair length greater than $10 \mathrm{~mm}$ (approximately $16 \mathrm{~mm}$ ) were also made (Supplementary Tables 1-5). The overall FFE of the N95 respirator was the most resistant to beard length, remaining at or above material specifications (i.e., $95 \%$ efficiency) at lengths up to $2.5 \mathrm{~mm}$, with moderate reductions in performance observed with increasing length out to $10 \mathrm{~mm}$ ( $\geq 80 \% \mathrm{FFE})$. The KF94 and KN95 masks showed a lower starting FFE compared to the N95 respirator, with the KF94 performing slightly better than the KN95 on average at all beard lengths tested (Supplementary Tables 1-5). However, both KF94 and KN95 masks showed proportionately similar decrements in overall FFE from their respective baselines as a function of beard length when compared to the N95 respirator. The plotted data support this observation, showing a rank order of overall FFE performance of N95 $>$ KF94 $>$ KN95 with increasing beard hair length up to $10 \mathrm{~mm}$ (Fig. 3) and beyond to approximately $16 \mathrm{~mm}$ (Supplementary Tables 1-5). The procedure mask and the cotton cloth mask overall FFEs were substantially lower than the N95 respirator, KF94, and KN95 at baseline and were notably unaffected by increasing beard length throughout the range tested (Figs. 2 and 3). For Volunteer 1, the slopes of linear regression analyses indicate the steepest negative impact on FFE for KN95 (-3.0), similar negative impacts for the KF94 and N95 (approximately -2 ), and values closer to zero for procedure and cloth masks. Data from Volunteer 2 showed similar results for KF94 (-2.4) and procedure masks $(0.1)$, but a slope closer to zero for the N95 respirator $(-0.1$, see Supplementary Fig. 1), suggesting a generalizable pattern for two of the three tested masks.

A previous report [9] showed that a significant improvement in FFP3 (European standard similar to N95) respirator filtration efficiency could be achieved in bearded individuals by covering the beard hair with an elastomeric band (e.g., a resistance exercise band). As shown in Table 2, when tested with $>8.5-\mathrm{mm}$ beard length, the band improved the FFE of the N95 respirator significantly, raising it above its rated performance level (i.e., $\geq 95 \%$ ). Use of the band also produced marked improvements in the FFE of the KF94 and KN95 masks (approximately 30 and 20\% improvements, respectively). The FFE of the procedure mask increased by a similar amount with the use of the band, while that of the cloth mask was raised only marginally (Table 2). Lower variance (SD) of obtained measurements, found for all except the cloth mask, further demonstrated the benefit of this intervention.

\section{Discussion}

Recent surveys found high percentages of British and American men $(42 \%$ and approximately $60 \%$, respectively) reported having or keeping a beard at least some of the time with even higher prevalence of facial hair among 18- to 39-year-old British men $(61 \%)$ [4, 5]. Given these rates of beard hair in the population, the findings of this study suggest a significant limitation on the efficacy of face masks as personal protective equipment against 
Fig. 2 Fitted filtration efficiency (FFE) percentage for face masks at different beard lengths measured using the Occupational Safety and Health Administration modified ambient aerosol CNC quantitative fit testing protocol. Data from Volunteer 1 show the overall FFE decreased for a NIOSH N95 respirator (A), a Korean standard KF94 mask (B), and a Chinese standard KN95 mask $(\mathbf{C})$, with increasing beard hair length $(0,1.5,3.0,6.0$, 9.0 , and $>10.0 \mathrm{~mm}$ ). The FFE percentages for a reusable cloth mask (D) and a procedure mask with elastic ear loops (E) were low even with shaven skin and did not deteriorate appreciably with beard hair length. The numbers adjacent to the data " $1,2,3$, and 4" indicate the starting time of the four exercises in the fit test, (i.e., $1=$ bending at the waist for $50 \mathrm{~s}, 2=$ reading aloud for $30 \mathrm{~s}, 3=$ looking left and right for $30 \mathrm{~s}$, and $4=$ looking up and down for $30 \mathrm{~s}$ ). Data corresponding to FFE of the face mask are expressed as [1 (mask count/ambient count)] $\times$ 100 shown as percent $(0-100)$ on the $y$-axis. The average of three independent tests is plotted against time on the $x$-axis (seconds), with 10 additional seconds recorded after each exercise.

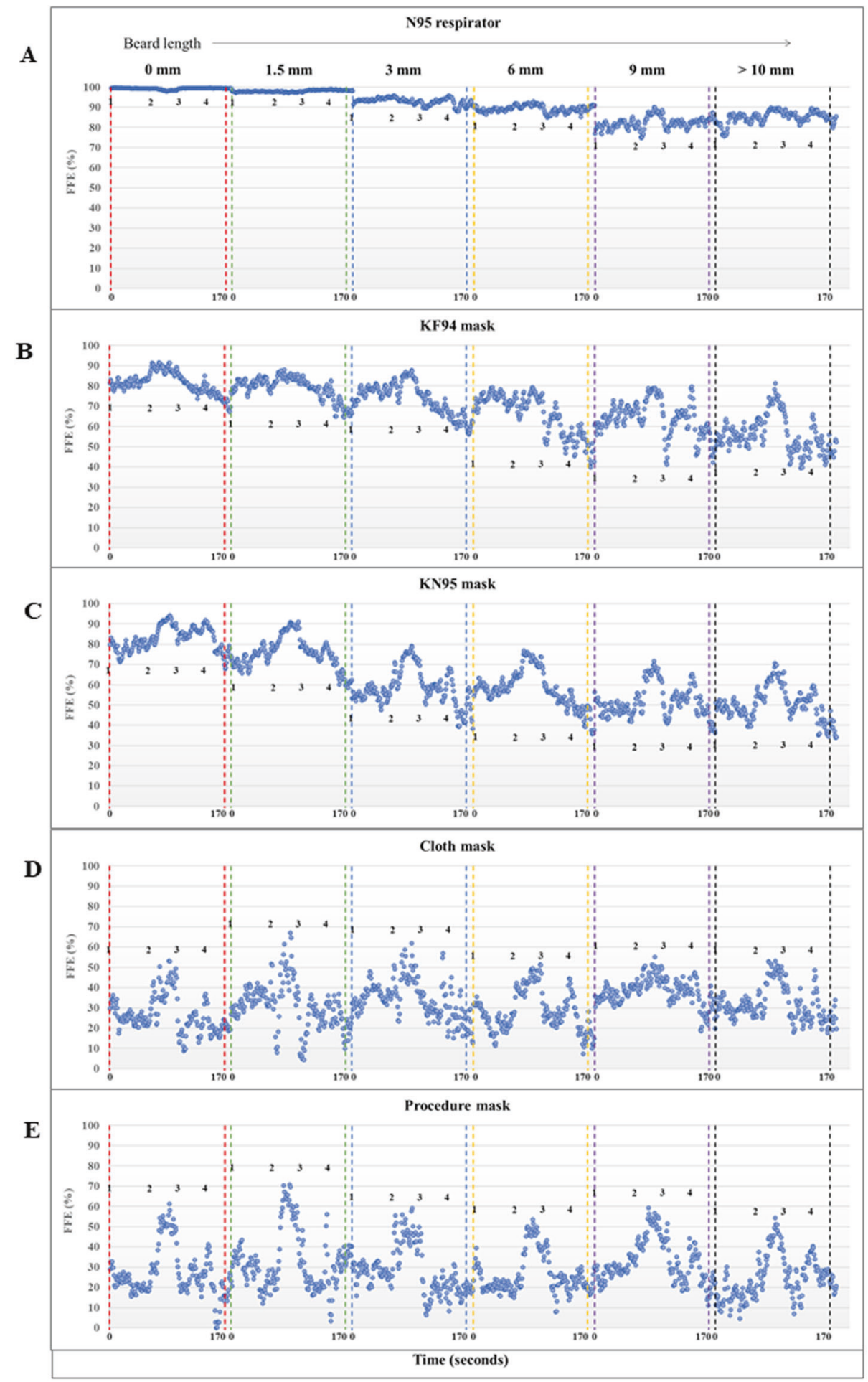

SARS-Cov-2 infection. Previous studies examining the effect of beard hair on the fit and performance of respirators have reported variable findings, with some showing significant impairment [10]. For instance, a recent survey of a cohort of Australian health care workers reported that no individuals with full beards could achieve a satisfactory respirator fit [11]. While other studies have shown satisfactory performance in bearded workers [12], the US OSHA prohibitions on respirator use and fitting include beard hair as a "condition that interferes with face-to-facepiece seal" [3]. The US Center for Disease Control and OSHA have published a chart showing recognized facial hair styles, most of which are contraindicated for respirator use [7]. 


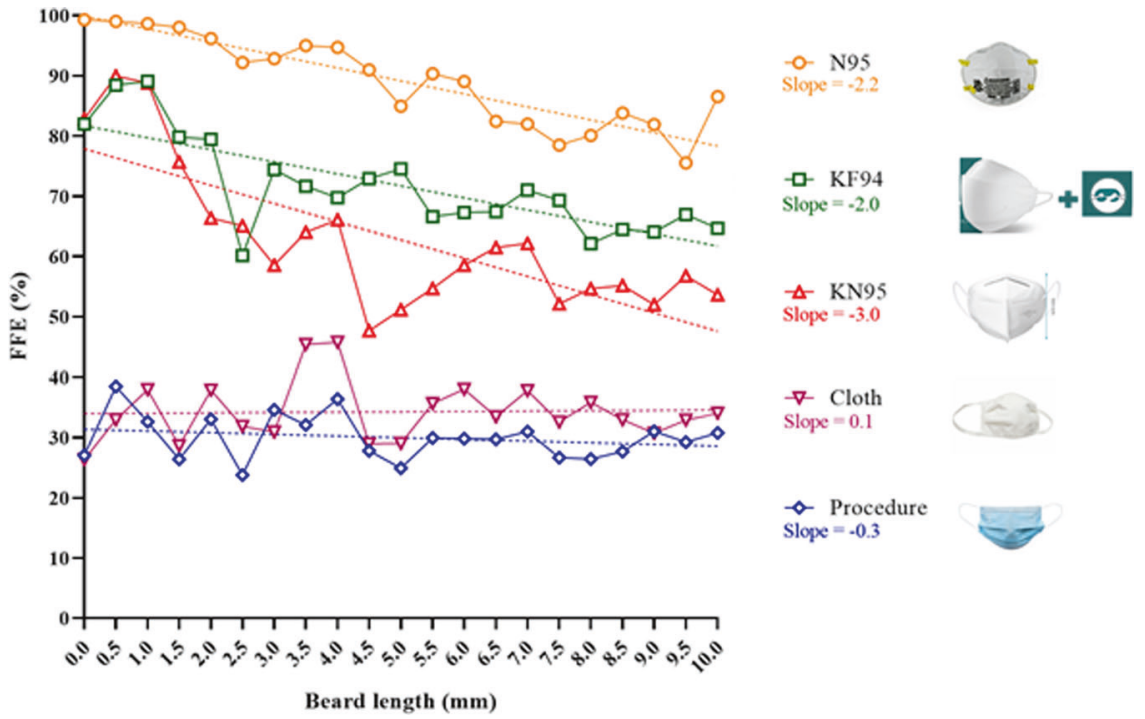

Fig. 3 The overall fitted filtration efficiency (FFE) for face masks at different beard lengths using the Occupational Safety and Health Administration modified ambient aerosol CNC quantitative fit testing protocol. The overall FFE as a function of beard hair length (data from Volunteer 1) in the range of $0-10.0 \mathrm{~mm}$ in $0.5 \mathrm{~mm}$ increments decreased for a N95 respirator (slope $=-2.2$ ), a Korean standard KF94 mask (slope $=-2.0$ ), and a Chinese standard KN95 mask (slope $=-3.0$ ). The comparatively low FFE of a procedure

Table 2 The effect of an elastic band worn over the beard on the fitted filtration efficiency of various face masks.

\begin{tabular}{lll}
\hline Mask (brand) & Yoga band & \% FFE (SD) $^{\mathrm{a}}$ \\
\hline N95 respirator (3M, 8210) & No & $85.3(3.1)$ \\
& Yes & $96.1(0.6)$ \\
& $\%$ improvement $^{\mathrm{b}}$ & +12.7 \\
KF94 micro-dust protection mask & No & $61.9(6.7)$ \\
(Dr Puri) & Yes & $80.2(3.8)$ \\
& $\%$ improvement $^{\mathrm{b}}$ & +29.6 \\
KN95 folding particle protection & No & $54.9(7.9)$ \\
disposable mask (Lei Shi De) & Yes & $65.7(5.7)$ \\
& $\%$ improvement $^{\mathrm{b}}$ & +19.8 \\
Procedure mask, earloop (Medline & No & $30.6(10.4)$ \\
Industries) & Yes & $36.6(7.7)$ \\
& $\%$ improvement $^{\mathrm{b}}$ & +19.7 \\
Cloth/cotton mask (Hanesbrands) & No & $39.4(7.3)$ \\
& Yes & $40.6(8.0)$ \\
& $\%$ improvement $^{\mathrm{b}}$ & +2.9 \\
\hline
\end{tabular}

${ }^{a}$ The efficiency (FFE) is calculated as [1-(mask count/ambient count) $\times 100$ across the length of the test. The FFE \pm SD are shown. The average of at least two unique tests is presented.

${ }^{\mathrm{b}} \%$ improvement was calculated as the percentage increase of FFE with and without the elastic band.

Information on the use of other types of face masks by individuals with beards is less clear than that available for respirators. A study by McLure et al. reported increased mask with elastic ear loops (slope $=-0.3$ ) and a reusable cloth mask (slope $=0.1)$ did not decrease further with beard hair length. The FFE is calculated as $[1-$ (mask count/ambient count) $] \times 100$ and presented as the average of all FFE data across the test period at each beard length. Linear regression was used to calculate the slope (dotted line) of FFE against beard lengths. The average of at least two independent tests is shown.

shedding of bacteria in bearded men wearing surgical masks relative to shaved individuals [13]. However, disposable surgical and procedure style masks are not subject to fit testing under OSHA regulations. Tests examining surgicalstyle masks have found 8-12 times higher total particle penetration compared to N95 respirators and attributed particle penetration to be driven in large part by facepiece seal leakage as opposed to filter media [14]. Recent demonstrations of improved procedure mask FFE following modification interventions support an important role of mask fit in enhancing their performance [15]. No guidelines are currently available in the US for Korean (KF94) and Chinese (KN95) face masks that are sometimes marketed to the public as high-efficiency alternatives to N95 respirators. Similarly, cloth face masks, a reusable, low cost, and environmentally friendly choice of respiratory protection, have no specific direction provided regarding their compatibility with beards.

The data shown in this study demonstrate that FFE decreased relatively linearly overall with beard length for N95 and KF94 masks, with a more variable decline observed for a KN95 mask. The N95 respirator showed the greatest tolerance of hair intrusion, with FFE remaining at or above $95 \%$ with beard length of $2.5 \mathrm{~mm}$, equivalent to approximately 7 days of growth for Volunteer 1 and consistent with "stubble" findings in a large study by the British Government [6]. Compared to the N95 respirator, the performance of KF94 and KN95 masks first showed FFE 
improvement with minimal stubble $(0.5$ and $1.0 \mathrm{~mm})$ followed largely by impairment with increasing beard lengths. An interesting possibility is that the gritty texture of stubble hairs may have facilitated a "Velcro effect" to enable the mask layer in direct contact with the face to adhere better and improve the seal with the skin. Beyond stubble growth, these masks, worn as recommended by the manufacturer, showed marked decreases in FFE with beard length, albeit the KF94 mask was consistently more resistant than the KN95 to increasing length throughout the range tested.

The decline in performance of higher efficiency respirator type masks as a function of increased beard hair growth is attributed to disruption of the seal formed between the margins of the face mask and the facial skin. From this perspective, the lack of a response to beard length shown by the procedure and cloth masks may reflect the overall low FFE performance of these face masks. Modest improvements in FFE within a narrow beard hair length range noted for Volunteer 1 could be related to facial morphology, suggesting these masks may have better conformed to the face with added depth. Other possibilities include added filtration efficiency by hair fibers, through sieving and/or electrostatic mechanisms, or an improvement in adherence of the face mask relative to bare skin. Improved FFE previously demonstrated with simple modifications to procedure earloop masks [15] suggests that such enhancements would also benefit bearded individuals.

As demonstrated here, covering beard hair with an elastic exercise band can substantially mitigate potential performance declines of face masks, by creating a surface that more closely approximates the facial skin. This low cost, easily available intervention is especially likely to benefit masks constructed with melt-blown materials that perform best when they fit well (good adherence and conformation to the face) and could be an option for bearded individuals for whom it is a cultural norm, religious imperative, or medical necessity (e.g., skin sensitivity) to not shave. Recent media reports [16] highlighted many males who have grown so-called quarantine beards. For this group, covering or closely trimming beards (combined with mask fit and seal enhancements), would likely provide a greater degree of protection from infectious aerosols.

Given the prevalent use of a wide variety of masks during the current pandemic, results from this study and previous work provide practical solutions to improve filtration performance across different types of populations as well as face masks. Evaluation and steps taken to match the shape, size, and features of faces with the best protective face masks broadly available, together with fit enhancements and training for proper wearing and usage, may help to reduce viral transmission. Future research on the relationship between facial features and FFE and the balance of improvement modifications as a function of material and fit parameters will help protect the health of wearers in the context of multiple airborne exposures including environmental pollution and pathogens such as the SARS-CoV-2 virus.

Although a partial replication was noted in data from Volunteer 2, one limitation of the current study is that to provide a controlled point of reference, most of the data showing the incremental effect of beard hair length on FFE of the five face masks tested and exercise bands were collected by repeated cycles of beard growth and fit testing of a single individual. Therefore, the findings may not completely capture the role of interindividual factors such as differences in facial morphology, beard density, and hair texture. Additional studies are needed to investigate the potential effect of these variables on face mask fitting and the potential interaction with beard hair. Higher overall FFE for bearded subjects wearing N95 respirators (Table 1 and Supplementary Fig. 1), relative to Volunteer 1, may be a function of the specific mask model tested, and/or characteristics of an individual's face and facial hair. Additional testing with different shapes, styles, and material rigidities of N95 respirators (e.g., horizontal shape with fold out flaps, cup shape, cone shape, duckbill) in a broader sample of volunteers would help to address the factors contributing to variability reported here and observed by others [6, 10-12]. Similarly, results we report here show the efficiency of face masks worn as personal protective equipment. The extent to which these findings are predictive of the performance of face masks as source control, specifically in reducing the emission of SARS-CoV-2 aerosols, is not known at present and is a focus of ongoing investigation. Finally, despite standardized procedures, individual differences in proficiency of mask fit testing can add variability to observed results.

Taken together, this study furthers our understanding of the impact of a variety of beard lengths on the efficiency of facial coverings commonly available to the public. Detailed and repeated results in a volunteer who showed impaired performance with facial hair indicated that FFE decreased linearly with hair length for the three most effective facial coverings tested (N95, KF94, and KN95). The relatively low performance of procedure style and cloth masks was not substantially impacted by the presence of facial hair. An intervention using exercise bands as a beard cover yielded performance improvements for all face masks tested, while reducing performance variability. Face mask performance is related to the materials used and the interaction of various shapes, sizes, and sealing surfaces with a given wearer's face. The presence of a beard is likely to impact fit parameters for many available styles and it is worth considering limiting its length, completely shaving (if feasible), or covering beards to achieve better filtration performance. Combining this approach with other readily available fit improvement modifications should help reduce exposure to 
environmental contaminant particles, including both pollution and pathogens such as the SARS-CoV-2 virus, and thereby benefit public health.

Acknowledgements The authors gratefully acknowledge Philip A. Bromberg, MD, and the journal reviewers for their critical review of the manuscript.

Author contributions SEP, HC, HT, and JMS had access to the data and take responsibility for the integrity of the data and the accuracy of the analysis. Concept and design: SEP and JMS. Data collection, analyses, and interpretation: SEP, JMS, HC, HT, WDB, KLZ, PWC, and JB. Drafting of the manuscript: SEP, HC, and JMS. Critical revision of the manuscript: all authors. Administrative, technical, or material support: SEP, JMS, HT. Supervision: JMS, HT, and SEP.

\section{Compliance with ethical standards}

Conflict of interest The authors declare no competing interests.

Publisher's note Springer Nature remains neutral with regard to jurisdictional claims in published maps and institutional affiliations.

\section{References}

1. Sunjaya AP, Jenkins C. Rationale for universal face masks in public against COVID-19. Respirology. 2020;25:678-9.

2. Oestenstad RK, Bartolucci AA. Factors affecting the location and shape of face seal leak sites on half-mask respirators. J Occup Environ Hyg. 2010;7:332-41.

3. OSHA Respiratory protection--OSHA. Final rule; request for comment on paperwork requirements. Fed Regist. 1998;63:1152-1300.

4. Statista. https://www.statista.com/statistics/725005/men-wearingbeards-us/. Accessed 18 Dec 2020.

5. Smith M. YouGov data shows how beards have become more popular since 2011, especially among younger Brits. 2017. https:// yougov.co.uk/topics/politics/articles-reports/2017/03/10/beards-a re-growing-british-public. Accessed 18 Dec 2020.
6. Frost S, Harding A. The effect of wearer stubble on the protection given by Filtering Facepieces Class 3 (FFO3) and Half Masks. Health and Safety Executive, 2015.

7. CDC. To Beard or not to Beard? That's a good Question! https:// blogs.cdc.gov/niosh-science-blog/2017/11/02/noshave/. Accessed 18 Dec 2020.

8. Sickbert-Bennett EE, Samet JM, Clapp PW, Chen H, Berntsen J, Zeman KL, et al. Filtration efficiency of hospital face mask alternatives available for use during the COVID-19 pandemic. JAMA Intern Med. 2020. https://doi.org/10.1001/jamainternmed. 2020.4221.

9. Singh R, Safri HS, Singh S, Ubhi BS, Singh G, Alg GS, et al. Under-mask beard cover (Singh Thattha technique) for donning respirator masks in COVID-19 patient care. J Hosp Infect. 2020;106:782-5.

10. Skretvedt OT, Loschiavo JG. Effect of facial hair on the face seal of negative-pressure respirators. Am Ind Hyg Assoc J. 1984;45: 63-66.

11. Sandaradura I, Goeman E, Pontivivo G, Fine E, Gray H, Kerr S, et al. A close shave? Performance of P2/N95 respirators in healthcare workers with facial hair: results of the BEARDS (BEnchmarking Adequate Respiratory DefenceS) study. J Hosp Infect. 2020;104:529-33.

12. Floyd EL, Henry JB, Johnson DL. Influence of facial hair length, coarseness, and areal density on seal leakage of a tight-fitting halfface respirator. J Occup Environ Hyg. 2018;15:334-40.

13. McLure HA, Mannam M, Talboys CA, Azadian BS, Yentis SM. The effect of facial hair and sex on the dispersal of bacteria below a masked subject. Anaesthesia. 2000;55:173-6.

14. Grinshpun SA, Haruta H, Eninger RM, Reponen T, McKay RT, Lee SA. Performance of an N95 filtering facepiece particulate respirator and a surgical mask during human breathing: two pathways for particle penetration. J Occup Environ Hyg. 2009;6: 593-603.

15. Clapp PW, Sickbert-Bennett EE, Samet JM, Berntsen J, Zeman KL, Anderson DJ, et al. Evaluation of cloth masks and modified procedure masks as personal protective equipment for the public during the COVID-19 pandemic. JAMA Intern Med. 2020. https://doi.org/10.1001/jamainternmed.2020.8168.

16. Lipstein RL. 2020. https://www.newyorker.com/culture/videodept/the-rise-of-the-quarantine-beard. 